\title{
Maxillary sinus diseases - diagnosis and differential diagnosis
}

\author{
Milena Petkova, Elitsa Deliverska \\ Department of Oral and Maxillofacial Surgery, Faculty \\ of Dental Medicine, Medical University, Sofia;
}

\begin{abstract}
The maxillary sinus is an airfilled cavity and is part of a series of paranasal sinuses and impacts most on the work of oral and maxillofacial surgeons as they will often be required to make a diagnosis in relation to inflammation, orofacial pain, tumour, etc. It could be mainly sinogenic or odontogenic in origin. Maxillary sinus diseases are often coincidentally observed on radiographs, and physicians often have to make a diagnosis and plan treatment based on the interpretation of the image. The purpose of this paper is to guide oral and maxillofacial surgeons and general practitioners through the etiology and processes of some of the diseases and clinical features involving the paranasal sinuses and in particular the maxillary sinus and to encourage comprehensive history taking and examination of the patient to facilitate an accurate diagnosis that will enable successful treatment.
\end{abstract}

Keywords: maxillary sinus, diseases, pathology, odontogenic

\section{Introduction}

The maxillary sinus is the largest among the paranasal sinuses. Diverse diseases may affect the maxillary sinus - including cystic, inflammatory or neoplastic lesions.

Our purpose was to make a review of the literature regarding the most common sinus pathologies. 


\section{Anatomy and physiology of maxillary sinus}

The paranasal sinuses are a group of air containing spaces present on either side in the skull (1). The paranasal sinuses include the maxillary, frontal, ethmoidal, and sphenoidal sinuses (2). The largest sinus is the maxillary sinus (1).

Maxillary sinuses can vary in size and shape from one individual to another, or even between the right and left sides in the same individual. In approximately half of the population, their length also varies (3).

The floor of the maxillary sinus extends into the alveolar process between the roots of adjacent teeth, creating elevations and depressions called "extensions," with narrow cortical areas (3). It has been radiographically demonstrated that most of the roots projecting into the maxillary sinus are in fact surrounded by a thin layer of cortical bone, with perforations present in $14-28 \%$ of cases. Under normal conditions, the relationship between the tooth and the floor of the maxillary sinus consists of either a thin layer of compact bone that provides support to the apical periodontal ligament fibers, to which it firmly adheres, or there is a direct relationship with the maxillary sinus mucosa (3). The bony wall, separating the maxillary sinus from teeth roots, varies from full absence, when teeth roots are covered only by mucous membrane, to a wall of $12 \mathrm{~mm}(4)$.

The inner lining of the maxillary sinus cavity is devoid of periosteum; therefore, in the absence of a thin layer of intervening bone, the periodontal tissues are in direct contact with the basal surface of the sinus mucosa. The roots of the upper premolars, molars, and occasionally the canine teeth have a close relationship with the maxillary sinus; in some cases, they may even protrude into it (3).

The membrane that lines the walls of the maxillary sinus is called Schneiderian membrane. It is multilayered and $0.13 \mathrm{~mm}$ to $0.5 \mathrm{~mm}$ in thickness (5). The paranasal sinuses mucosa exhibits specific features: it is much thinner and composed of ciliated and nonciliated pseudostratified columnar epithelium. The cilia act in mass, producing coordinated sequential beating and thus, creating a wave-like motion, generally in the direction of the ostium (6).

The maxillary sinus drains via the ostia situated in the medial wall in the middle meatus. About 4 to $5 \%$ have accessory ostia (1).

This sinus has relationship with the oral cavity, nasal cavity, orbital cavity and infratemporal fossa.

Functions of maxillary sinus

- $\quad$ 1- speech and voice resonance

- $\quad 2$ - reducing the weight of the skull

- 3- warmth inhaled oxygen

- 4- filtration of the inspired air

- 5- immunological barrier

- 6- regulation of intra nasal pressure

- 7-protection the skull base from trauma. 


\section{Classification of the diseases of maxillary sinus (tabl.1)}

Table 1. Diseases involving maxillary sinus

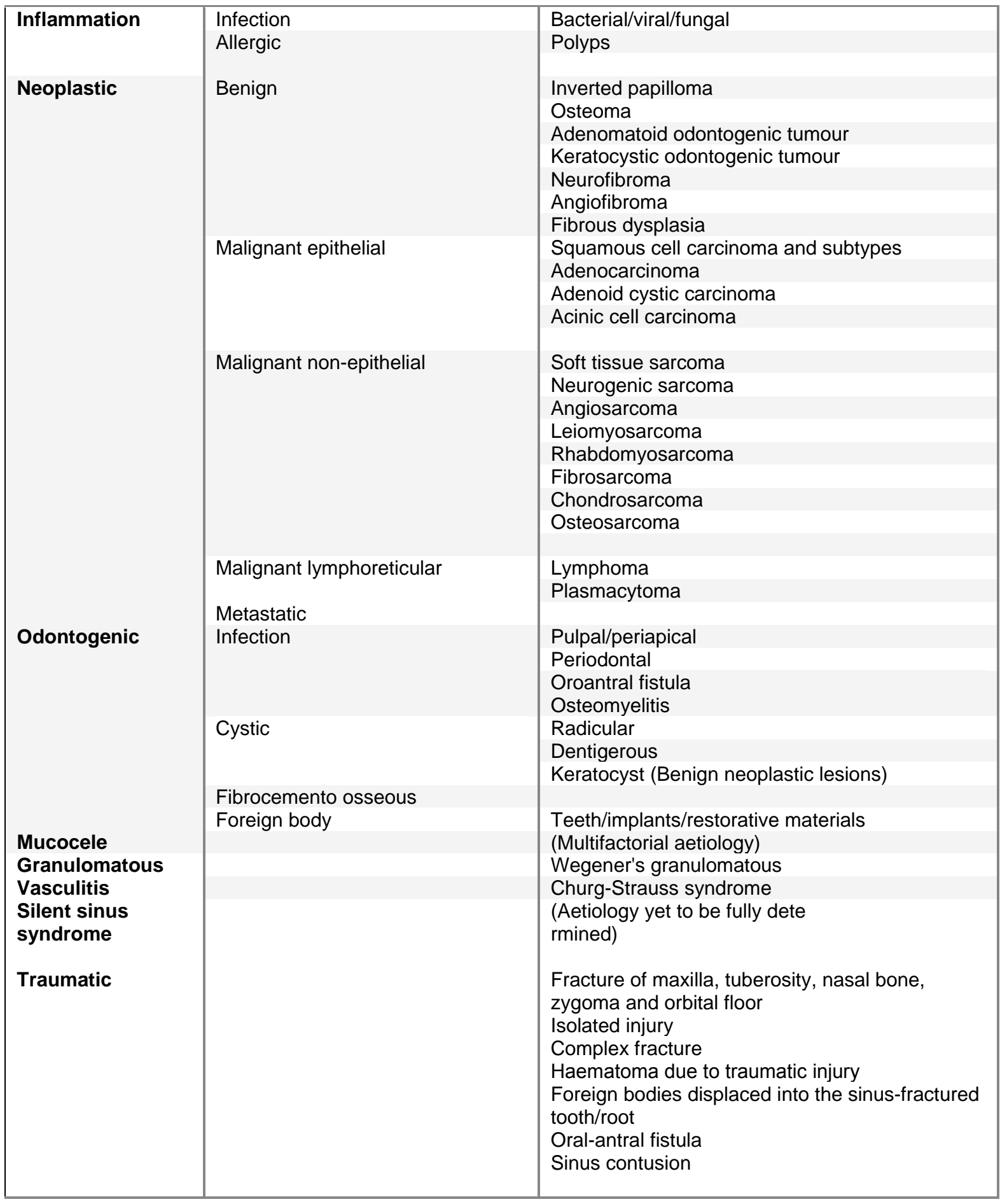


Maxillary sinuses mainly (Table 1) can host cystic, inflammatory or neoplastic lesions (7).

\section{Inflammatory diseases}

Inflammation of the mucosa of the paranasal sinuses is referred to as sinusitis. When the maxillary sinus is involved, it is called maxillary sinusitis. Most of the sinusitis are rhinogenic in origin due to viral, bacterial or fungal infections. They cause edema of the nasal mucosa and obstruct the drainage of the sinuses (1). Other causes can be anatomical obstruction of the sinus by deviated nasal septum, allergic rhino-sinusitis, post traumatic sinusitis, or mucociliary clearance disorders such as Kartagener's syndrome, Young syndrome, and infections of odontogenic origin. General immunosuppressive status like uncontrolled diabetes mellitus, acquired immune suppressive disorder and others are common predisposing and aggravating factors (1).

\section{Etiology of maxillary sinusitis}

Maxillary sinusitis of odontogenic origin (fig.1) is a well-known condition in both the dental and otolaryngological communities. It occurs when the Schneiderian membrane is violated by conditions arising from a dentoalveolar unit (4). This type of sinusitis differs in its pathophysiology, microbiology, diagnostics and management from sinusitis by other causes, therefore, failure to accurately identify a dental cause in these patients usually leads to persistent symptomatology and failure of medical and surgical therapies directed toward the sinusitis (4).

Fig 1. Maxillary sinusitis of odontogenic origin (unilateral, periapical infection, periodontal disease, tooth extr., foreingh body, sinus exposure, etc.

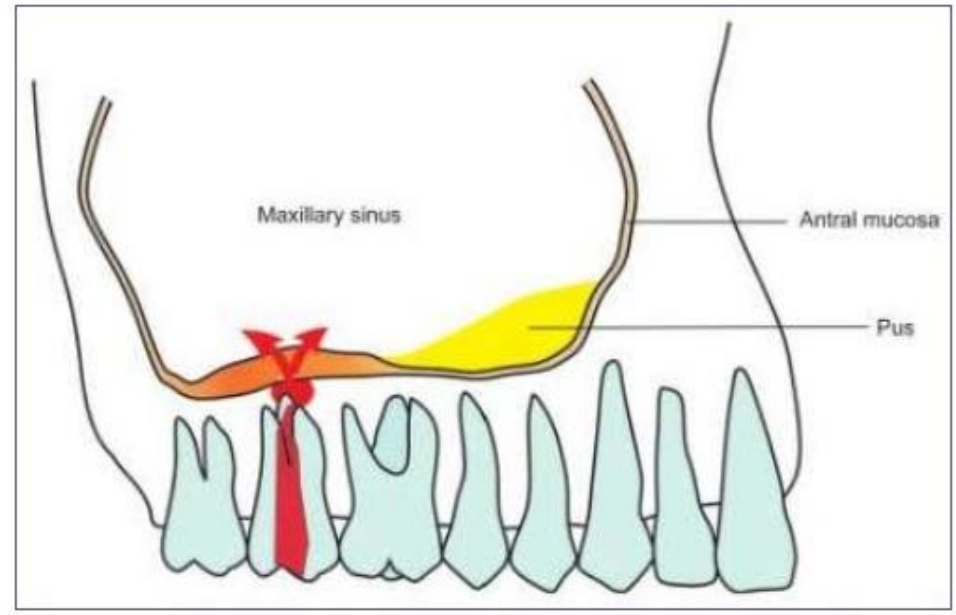

Multiplication of bacteria invading from the focus of a dental infection results in ndentooenis maxillarv cinusitis

Dental pathology is considered to be the etiologic factor in $10 \%$ to $12 \%$ of the maxillary sinusitis cases ( 1 , $4,6)$. However, in recent publications, up to $30-40 \%$ of chronic maxillary sinusitis cases are attributed to a dental cause (4). Intimate anatomical relation of the upper teeth to the maxillary sinus promotes the development of periapical or periodontal odontogenic infection into the maxillary sinus $(3,4)$. Hence, Eberhardt conducted a study with CT in which he measured the distances between the maxillary sinus floor and the tips of the posterior maxillary molars, finding that the tips of the molars were generally closer to the sinus than the premolars, especially the mesiobuccal root of the second upper molar. Other authors emphasize that depending on the degree of sinus pneumatization, the roots can be projected into it, even 
some of the tips can become coated by the sinus mucosa. In other studies using CT, similar results were obtained regarding the distance between the roots and the sinus, and it was found that a small percentage of patients had roots that extended into the walls of the sinus (8). It has been demonstrated that, in cases of apical periodontitis, when the tip of the tooth root was in contact with the floor of the maxillary sinus, the incidence of mucosal thickening was lower than when the tip of the root exceeded the floor of the maxillary sinus, so the closer the tooth apex is to the floor of the maxillary sinus, the greater the impact on antral tissue (3). Of the odontogenic sinus diseases, apical periodontitis and periodontal disease account for $83 \%$ of all cases having dental origin (3). In a metaanalysis made by Arias-Irimia the most common cause of odontogenic maxillary sinusitis were iatrogenic causes. (55.97\%). Other possible etiologies according this study were periodontitis (40.38\%) and odontogenic cysts (6.66\%). Oroantral fistulas and the remaining roots, taken together as iatrogenia after tooth extraction, accounted for $47.56 \%$ within iatrogenic causes. The dressings to close these oroantral fistulas and non-specific foreign bodies for the $19.72 \%$, extrusion of endodontic obturation materials into the maxillary sinus represented $22.27 \%$, amalgam remains after apicoectomies $5.33 \%$, maxillary sinus lift preimplantology surgery $4.17 \%$, and poorly positioned dental implants or those migrated to the maxillary sinus $0.92 \%$ of all cases included under an iatrogenic source of infection. On the other hand, Lee and Lee made a retrospective chart analysis of 27 patients with OMS and found that implant related causes were most common, which accounted for $37 \%$ of the cases. Dental extraction-related complications were the second most common cause according to this study and were found in $29.6 \%$ of cases. A follicular cyst was seen in $11.1 \%$, a radicular cyst, dental caries complications, and a supernumenary tooth were each found in $7.4 \%$ of cases. About the main tooth involved, the molar region stood out with a maxillary sinusitis ocurrency of $47,68 \%$. The first molar was the most frequently affected one with an incidence of $22.51 \%$, followed by the third molar $(17.21 \%)$ and the second molar tooth (3.97\%). Regarding the premolar region, it was only affected in $5.96 \%$ of the cases, being the second most frequently involved premolar (1.98\%). The canine only participated in $0.66 \%$ of the cases of maxillary sinusitis (4).

\section{Microbiology}

The typical odontogenic infection is now considered to be a mixed aerobic-anaerobic infection, with the latter outnumbering the aerobic species involved. The most common organisms include anaerobic streptococci, Bacteroides, Proteus, and Coliform bacilli (2) The higher frequent recovery of anaerobes in sinusitis is associated with an odontogenic origin that may be related to the poor drainage and increased intranasal pressure that develops during inflammation. This can reduce the oxygen tension in the inflamed sinus by decreasing the mucosal blood flow and depressing the ciliary action. The lowering of the sinus cavity's oxygen content and $\mathrm{pH}$ supports the growth of anaerobes $(6,9)$. Itzaket et al.'s study on the microbiology of acute sinusitis showed the predominant aerobic organisms to be a-hemolystreptococci, microaerophilic streptococci, and Staphylococcus aureus. The predominant anaerobes were anaerobic Gram-negative bacilli, Peptostreptococcus, and Fusobacterium spp. In chronic sinusitis, aerobes were recovered in $11 \%$, anaerobes only in $39 \%$, and mixed aerobic and anaerobic bacteria in $50 \%$. The predominant aerobes were a-hemolytic streptococci, microaerophilic streptococci, and S. aureus. The predominant anaerobes were Gram-negative bacilli, Peptostreptococcus and Fusobacterium spp. $\beta-$ lactamase-producing bacteria (BLPB) were recovered from $50 \%$ of the patients with acute sinusitis and from $75 \%$ of patients with chronic sinusitis. Fungal infections like Aspergillosis and Mucormycosis are common in immunocompromised individuals, but can also occur in non-immunocompromised ones (1).

The sinus mucosa is considered thickened when the membrane is 2- $6 \mathrm{~mm}$ thick. The etiological factors are related to some type of irritation (3). The presence of a foreign body in the sinusal cavity, for example, represent an irritation factor for the sinonasal mucosa and can cause marked structural reorganization of the mucous membrane usually with the predominance of hypertrophic and polypous changes. Besides these changes, the presence of a foreign body of dental origin, especially root canal fillings, are well known factors for the development of a fungal infection (like fungus ball) of the maxillary sinus. This is due to the fact that, because of chronic sinusal mucosal changes, in the sinus cavity can appear small nuclei on which salts can precipitate and form anthroliths, cysts or fungal sinusitis especially caused by 
Aspergillum spp. (10). Mucosal thickening of the maxillary sinus is a normal finding in asymptomatic patients, but a thickening greater than $2 \mathrm{~mm}$ can be associated with maxillary sinusitis (3).

\section{Signs and symptoms}

In 1996, the American Academy of Otolaryngology-Head and Neck Surgery established parameters for distinguishing the subtypes of sinusitis. Signs and symptoms associated with sinusitis are divided into major and minor groups. The classification is based on the duration of the disease and the presence of a specified combination of signs or symptoms determined by history and physical examination (11).

Acute sinusitis presents as facial pain over the maxillary sinus area, nasal obstruction, nasal discharge, dental pain, general constituitional symptoms like fever, malaise, headache, etc. Signs of acute sinusitis are tenderness over canine fossa, congested odematous nasal mucosa, since the nasal and systemic symptoms predominate dental etiology may be missed (1). These symptoms should have less than three weeks duration (11).

Chronic sinusitis is defined as signs and symptoms of sinusitis which persist for more than 12 weeks, four episodes of acute sinusitis of greater than 10 days duration per year, or both(11). Chronic odontogenic maxillary sinusitis presents as chronic nasal obstruction unilaterally or bilaterally, purulent nasal discharge, decreased smell or foul smelling nasal discharge, post nasal drip, specific dental pain, bleeding while brushing teeth, etc. Signs of chronic maxillary sinusitis are pus in middle meatus (detectable by anterior rhinoscopy), chronic nasal mucosal congestion, and postnasal drip. Dental signs can be periodontitis, alveolitis, loose tooth with dental abscess, osteomyelitis, etc. (1).

Classic symptoms suggestive of an odontogenic source can include sinonasal symptoms such as unilateral nasal obstruction, rhinorrhea, and/or foul odor and taste. Brook adds such symptoms as headaches, unilateral anterior maxillary tenderness and postnasal drip. Dental symptoms, such as pain and dental hypersensitivity, do not reliably predict an odontogenic cause. The infrequency of dental complaints may be due to preserved patency of the osteomeatal complex of the maxillary sinus, which allows egress of pressure from within the sinus. In a case series of 21 patients with odontogenic sinusitis, dental pain was present in only $29 \%$ of the patients. These findings highlight the importance of maintaining a high level of suspicion for an odontogenic source of infection even in the absence of dental pain. Upper dental pain may also reflect primary sinusitis with referred pain to the teeth. Sinonasal symptoms predominate in patients with odontogenic sinusitis; however, these symptoms do not distinguish odontogenic sinusitis from other causes of sinusitis. Furthermore, no single symptom from the various sinonasal complaints associated with sinusitis has been shown to predominate in odontogenic sinusitis. In a retrospective chart review of 27 patients diagnosed with odontogenic sinusitis, Lee and Lee reported that unilateral purulent rhinorrhea was most common and found in $66.7 \%$ of their patients with OMS, followed by ipsilateral cheek pain in one-third of the patients, whereas $26 \%$ reported a foul smell or taste. The case series by Longhini reports unilateral nasal obstruction as the most common and bothersome symptom followed by facial pressure/pain. Therefore, unilateral sinus disease associated with a rotten or foul taste appears to be the only clinical finding most likely to differentiate between nonodontogenic sinusitis and odontogenic sinusitis (4).

An odontogenic maxillary sinusitis, fungal or bacterial, can very easily spread to involve other sinuses and ultimately cause a pansinusitis. Due to the direct anatomic relation between the orbit and paranasal sinuses, orbital complications are not uncommon. Extension of infection intracranially can occur by the following mechanisms : (a) direct extension - erosion through the posterior wall of the frontal sinus or skull base; and (b) indirect extension - by thrombophlebitis or septic embolism via the intracranial/extracranial valveless connecting veins (10). The infection may spread from the involved sinus to the central nervous system where it can cause serious complications such as subdural empyema, brain abscess, or meningitis (9). 


\section{Diagnosis}

The accurate diagnosis of odontogenic maxillary sinusitis (OMS) is particularly important, because its pathophysiology, microbiology and treatment differ from those of other forms of maxillary sinusitis (4).

\section{Radiographs}

The panoramic radiograph is a standard radiograph used in dental offices. This view is useful for evaluating the relationship of the maxillary dentition to the sinus, pneumatization, and pseudocysts. The overlap of the hard palate limits the usefulness of this examination for thorough evaluation. A panoramic radiograph is more useful for identifying displaced roots, teeth, or foreign bodies in the sinus. It gives detailed informaion about the lower part of the sinus. Dental examinations also include plain radiographs to evaluate for dental and/or periodontal disease. However, these dental radiographs have been shown to have estimated sensitivity of $60 \%$ for caries and approximately $85 \%$ for periodontal disease, leaving a high false negative rate (4). CT is the gold standard in the diagnosis of maxillary sinus disease due to its high resolution and ability to discern bone and soft tissue. Case series by Patel revealed that all patients with odontogenic sinusitis showed signs of dental disease on CT scan, with $95 \%$ of patients showing periapical abscesses on CT (4). CT requirement must be considered in patients with persistent maxillary sinus symptoms, in order to identify an odontogenic reason for the sinusitis (6). Cone beam CT is a relatively new informative tool which utilizes approximately $10 \%$ of the radiation dose of the conventional thin-slice $\mathrm{CT}$, and is able to image bony detail exquisitely, although soft tissue detail is reduced. The technique is gaining popularity among dentists (4). The main advantages of the method are high resolution of the images and low radiation dose. The images allow informative evaluation of the pathology of the maxillary sinus and posterior lateral teeth and their possible mutual connection (12).

Biopsy- FNAB, incisional, excisional

Microbiological examination for cultural sensitivity test

\section{Treatment plan}

The treatment of odontogenic origin sinusitis includes the abolition of the dental underlying factors and the management of the maxillary sinus infection.( 6) Sometimes dental treatment alone is adequate to resolve odontogenic sinusitis and sometimes concomitant or subsequent functional endoscopic sinus surgery or Caldwell-Luc operation is required (4).

Although odontogenic therapy and surgical drainage are of primary importance, antimicrobial therapy is an essential part of the management of serious odontogenic infections and their complications (9). Antibiotics are mandatory for 14-21 days (6).

\section{Antroliths}

The maxillary sinus can host antroliths. An antrolith is a calcified mass within the maxillary sinus. Stones arising in the antral cavities are uncommon, and their development is similar to that of a sialolith. The origin of the nidus of calcification may be extrinsic (foreign body in sinus) or intrinsic (stagnant mucus, fungal ball, bony fragments, blood and pus). Smaller antroliths are usually asymptomatic and may be discovered incidentally on routine radiography of the region. Larger ones may present as sinusitis with symptoms like pain and discharge $(13,14)$.

However, the majority of patients with maxillary antroliths in the literature have symptoms or clinical signs that may include pain, nasal stuffiness and obstruction, epistaxis, foul intraoral discharge, postnasal drip, tenderness over the involved sinus and, oro-antral fistula, foul-smelling discharge, facial pain, sinusitis (15). 
Radiographically, a dense, irregular yet well-defined mass can be identified in the antrum. They can be seen on panoramic, periapical, and Waters' radiographs in addition to computed tomographs (13).

Histologically, antroliths usually show concentric rings like those seen in stones found in other parts of the body. Chemical analyses show that these calculi contain various amounts of calcium phosphate, calcium carbonate, calcium oxalate, albuminous material, magnesium phosphate, organic matter and water. The consistency varies from hard and friable to soft, porous, or crumbly. Stones are frequently covered with granulation tissue with a rich blood supply. Color varies from black to gray, brown or white (15).

Antroliths must be included in the differential diagnosis of radiopacities found in or near the maxillary sinus region (13).

Clinical differential diagnosis of antral radiopaque areas should include displaced or ectopic tooth fragments, calcified mucous retention cyst, mycoliths, antroliths, cementoma, calcified polyps, osteoma, osteoblastoma, odontoma, rhinoliths, bone cysts, primary or metastatic carcinoma, osteogenic sarcoma, fibrous dysplasia, calcifying epithelial odontogenic tumor, fungal infection with secondary calcification, and foreign bodies (15).

The management of antroliths should include surgical removal of stone by an endoscopic sinus surgery with or without Caldwell-Luc operation, along with appropriate treatment of sinus infection (13).

Diverse disease entities may cause masses in the maxilla. Malignant tumors of the maxillary sinus and benign conditions including fibroosseous disease, mucocele, and various odontogenic cystic lesions may present with expansile maxillary masses (16).

With computed tomography (CT), solid tumor or fibroosseous disease can be easily distinguished from a cystic lesion, which shows homogeneously low attenuation with frequent remodeling of bony walls. The exact site of origin is very important in the differential diagnosis. A lesion of antral origin is likely to be a maxillary mucocele with various possible causes; for extraantral maxillary cystic lesions, the probable diagnoses are fissural cysts, cystic tumors of the maxilla, or cystic masses of dental origin (16).

\section{Mucous retention cyst}

The mucous retention cyst of the maxillary sinus (MRCMS) is a benign and self-limiting lesion, resulting from the leakage of mucus within the sinus mucosa due to ductal obstruction. It has been suggested that the mucous retention cyst is of non-odontogenic origin, since it may also occur in dentate and edentulous patients. Nevertheless, Veltrini et al. emphasized that periapical and periodontal disease associated with maxillary molars may be an important etiological factor for sinusitis and mucous retention cyst (17).

Mucosal cysts of the maxillary sinus (MMC) are common findings in imaging studies. Their prevalence is found to be up to $35.6 \%$ (18). Although several studies suggest that MRCs are found in the greatest proportion in the second and third decades of life, there have been reports of the highest frequency found beginning in the fourth and even sixth decade of life (7). For some authors, the frequency of MRCs may vary according to environmental factors, such as the seasons of the year, the mean temperature and the relative air humidity (7). Females and those aged 18-35 years were the groups with the greatest number of cases. The peak frequency was in August, and the lowest was in February. Statistical analyses revealed a correlation between the frequency of MRCs and the mean temperature (7).

Schuknecht and Lindsay described 2 types of maxillary sinus cysts and referred to them as nonsecreting or secreting cysts. The latter were classified as retention (glandular) cysts or mucoceles. Gardner modified this classification into nonsecreting cysts, secreting cysts (retention cysts), and mucoceles. The nonsecreting cysts (also referred to as "pseudocysts") are not lined with epithelium. They are characterized by a very thin membrane with an inner layer of compressed connective tissue cells and are 
suspected to arise in the subepithelial connective tissue due to a retention of fluid. The secretory or retention cysts are cystic formations due to obstruction of the mucosal glands and are less common than the nonsecreting cysts. The mechanism of their formation could possibly be a plug of inspissated mucus of the duct of the seromucinous gland of the sinus, which results in dilatation of the duct into a cystic structure. The secretory cysts are lined by epithelium. Various etiologic factors like allergy, barotrauma, rhinitis, dental disease, inflammatory process, and chronic rhinosinusitis have been proposed (18).

Mucous retention cysts are usually asymptomatic, seldom require treatment and are diagnosed with routine radiographic examinations. Clinically, they may become significant when they promote the obstruction of the maxillary sinus outflow tract, when they occur with symptoms or when the diagnosis is in doubt (7).

The MMC are defined, in terms of a panoramic radiograph or Water's view radiograph, as rounded domeshaped shadows originating from the wall or floor of the sinus. Definition per computed tomography (CT) scan includes the following criteria: 1 . Homogenous dome-shaped cysts with sharp demarcation of the lateral border; 2. Absence of bone destruction; 3. Absence of communication with tooth roots (to exclude odontogenic cysts); 4. A smooth, spherical outline along the free border of the cysts. (18) In cases in which the retention cyst completely fills the maxillary sinus, the radiographic interpretation becomes difficult, and complete blurring can be interpreted as maxillary sinusitis (17).

MRC have to be differentiated from several types of sinus diseases, such as sinusitis, mucocele, polyps, inverting papilloma, chronic rhinosinusitis with nasal polyps, or mycetoma, benign and malignant neoplasm, and odontogenic cyst. Polyps are less common lesions, which have been associated with allergic processes. Furthermore, polyps are associated with mucous thickening and are multiple lesions while MRC are solitary ones. Malignant tumors are usually asymmetrical or have irregular contours. These lesions promote the destruction of the sinus wall, mainly the posterior, and are accompanied by paresthesia. One finding that is some help in distinguishing odontogenic cyst from MRCs is the lack of a delineating radiopaque border in the latter because it originates within soft tissues outside the maxillary bone $(7,18)$. The differentiation of MMC from other unilateral maxillary sinus opacification is important in order to perform proper management (18).

Symptoms that have been related to MMCs include headache, nasal obstruction, facial pain, postnasal drip, and nasal discharge. Additionally, MMC were suspected to be a focus in patients with general fatigue, dizziness, fever of unknown origin, or arthritis. Patients with MMC were reported to have a respiratory allergy in $18 \%$ to $83.5 \%$ and sinonasal symptoms in $10 \%$ to $67.5 \%$.

Additionally, a sudden gushing of a yellowish fluid from the nostrils in the case of a spontaneous rupture can be directly related to MMC. A symptom that could be related to MMC in individual cases is also the development of an antrochoanal polyp. On the other hand, recent prospective comparative studies showed no correlation of sinonasal symptoms with MMC (18). Symptomatic cases may occur when the sinus is completely full, the most common clinical manifestations being that of headaches, periorbital and hemifacial pains, and sometimes dizziness, nasal obstruction and recurrent sinusitis, among others (8).

Most papers conclude that that majority of the patients with MMC do not need any treatment especially when the lesion is small and asymptomatic. Kanagalingam et al. stated that surgery in the sense of extracting $\mathrm{MMC}$ is mostly unnecessary because the cysts do not reflect sinonasal disease (18). Only radiographic monitoring is recommended, since most do not evolve and some regress spontaneously. In larger or symptomatic lesions, treatment should be performed by enucleation or curettage (17). Only patients with complaints should be treated. Before endonasal endoscopic sinus surgery, transoral approaches with or without Caldwell-Luc operation or puncture and cyst aspiration through the inferior nasal meatus have to be performed (18).

A mucocele is an expansion of the sinus cavity caused by an obstruction of the sinus ostium and an accumulation of secretion. This is the most significant local complication of sinusitis as a result of 
obstruction of the sinus ostium. As an antral lesion, a mucocele may cause expansion or erosion of the maxilla. A mucocele formation with inflammatory ostial obstruction in the maxillary sinus is uncommon. Mucoceles on rare occasions can occur within septated compartments in the maxillary sinus. In these cases, only a portion of the sinus is expanded and there may still be residual areas of erosion. Mucoceles also occur in the maxillary sinus after Caldwell-Luc procedures. They are thought to be a late complication after Caldwell-Luc procedure and are caused by a secretion from residual epithelium in postoperative maxillary sinuses. The most frequent site of bone defect and soft tissue bulging is the anterior wall in which the bone windows were made during Caldwell- Luc procedures (16).

Extraantral lesions include a number of benign odontogenic cystic lesions and fissural cysts. Odontogenic cystic lesions include developmental odontogenic cysts such as primordial and dentigerous cysts, odontogenic keratocysts, residual cysts, cystic ameloblastomas, and inflammatory lesions such as radicular or periapical cysts. Histologic analysis of the epithelial layers, association with other clinical findings, relationship to a tooth, and internal calcification allow further subdivision (16).

All nontumorous odontogenic cysts show similar radiologic findings of clearly demarcated expansile cystic mass, and are related to the root or crown of a tooth. Occasionally odontogenic cysts develop in the maxilla and extend into the antral cavity (16).

Because these fissural cysts and odontogenic lesions arise within the maxillary alveolar process, it is not surprising that cases with extraantral lesions show bony plates between the cystic lesion and the maxillary antrum. These bony plates represent the displaced and remodeled floor of the maxillary sinus. The alveolar process has cancellous bone between two layers of cortical bone. Many odontogenic lesions arise within the alveolar process and so expand the floor of the maxillary sinus upward (16).

By identifying the bony plate between the cystic mass and the maxillary antrum, one can distinguish an extraantral cystic lesion from an antral lesion such as a mucocele. However, a mucocele arising in a septated compartment of the maxillary sinus may mimic an extraantral lesion by showing a thin bony wall between the lesion and the adjacent antral cavity (16).

Because an observation of the displaced cortical plate is important, CT would be the most useful imaging mode for these evaluations (16).

The nasal cavity and paranasal sinuses are lined by a layer of mucus-producing tissue with the following cell types: squamous epithelial cells, minor salivary gland cells, nerve cells, infection-fighting cells, and blood vessel cells. Different types of cells in the paranasal sinus may become malignant. That is why a variety of tumor types may be discovered in the maxillary sinus including squamous cell carcinoma, adenocarcinoma, malignant melanoma, lymphoma, and sarcoma.

Tumours of the paranasal sinuses account for approximately $3 \%$ of all head and neck malignancies. Carcinomas arising in the maxillary sinus comprise $80 \%$ of this group of tumors. The majority of maxillary sinus carcinomas are locally advanced at diagnosis because their symptoms are nonspecific and resemble sinusitis. Spread to regional lymph nodes at initial presentation is evident in approximately $15 \%$ of cases. Treatment of these tumors has included either surgery, radiation therapy, or a combination of both (19).

Sinonasal tumors have a poor survival, despite early diagnosis, radical surgical resection, and strict followup (20).

Oral SCC is a disease entity with well established risk factors. It can be present on the tongue, floor of the mouth, buccal mucosa, or gingiva. Oral SCC slowly invade the underlying tissues after onset. Invasive maxillary SCC will exhibit a multitude of clinical signs and symptoms, which might mimic facial pain syndromes, including TN. In the early stages of the disease, the patient will first complain of localized maxillary pain; later, symptoms might progress to mobility of teeth. As the tumour invades the maxilla and 
infiltrates the maxillary sinus at a second stage, the patient will experience nasal congestion due to directobstruction symptoms. The infraorbital nerve (branch of the maxillary division of the trigeminal nerve) will also be affected, resulting in sensory disturbances of the cheek (21).

In differential diagnosis consideration should be included also of injuries that affect the maxillary sinus:

- $\quad$ Fracture of maxilla, tuberosity, nasal bone, zygoma and orbital floor

- Isolated injury

- $\quad$ Complex fracture

- Hahematoma due to traumatic injury

- $\quad$ Foreign bodies displaced into the sinus-fractured tooth/root

- $\quad$ Oral-antral fistula

- $\quad$ Sinus contusion

\section{Conclusion}

Unilateral maxillary sinus pathology is a relatively common finding. Early identification of a disease should avoid delay in surgical intervention, whereas some of the diseases can initially be treated medically. Careful history, clinical, radiographic and endoscopic examination can often determine the appropriate diagnosis and treatment.

\section{References}

1. Colbert KAR, Devakumari, Sankar R. Odontogenic Maxillary Sinusitis -Need for Multidisciplinary Approach-A Review. IOSR Journal of Dental and Medical Sciences (IOSRJDMS).Volume 13, Issue 6 Ver. III (Jun. 2014), 25-30.

2. Mehra $\mathrm{P}$, Murad $\mathrm{H}$, Maxillary sinus disease of odontogenic origin. Otolaryngologic clinics April 2004.Volume 37, Issue 2, Pages 347-364.

3. Roque-Torresa GD., Ramirez-Soteloa LR, Azevedo Vazb SL, Bóscoloa SMA, Bóscoloa FN. Association between maxillary sinus pathologies and healthy teeth. Braz J Otorhinolaryngol. 2016;82(1):33-38.

4. Simuntis R, Kubilius R, Vaitkus S. Odontogenic maxillary sinusitis: A review. Stomatologija, Baltic Dental and Maxillofacial Journal, 2014, Vol. 16, No. 2, 39.

5. Lindhe J, Lang N, Karring T. Ch. 50: Elevation of the maxillary sinus floor. Clinical Periodontology and Implant Dentistry, Fifth Edition 2008.

6. Pereiraal, Vaz P, Ricardo F. Almeidaa, Purificac, Tavares, Borsattoc C, Felino A. Genetic susceptibility to maxillary sinus pathology development in the presence of an odontogenic cyst. rev porte stomatolmeddentcir maxilofac. 2014; 55(2):110-114. 
7. Vieira EM., Vieira EM., de Morais S., Musis CR., Borges Á,H., Palma VC., Da Silva L., Guedes OA. Frequency of Maxillary Sinus Mucous Retention Cysts in a Central Brazilian Population. J Dent Shiraz Univ Med Sci., September 2015; 16(3): 169-174.

8. Martinez-Gonzalez JM, Barona-Dorado C, Arias-Irimia O, Martinez-Rodriguez N, FernandezDominguez M. Panoramic and tomographic implant studies: role in the diagnosis of sinus disorders. Med Oral Patol Oral Cir Bucal. 2010; 15: e611-e615.

9. Brook I. Microbiology of acute and chronic maxillary sinusitis associated with an odontogenic origin. Laryngoscope. 2005;115:823-825.

10. Manea $C$, Sarafoleanu C.latrogenic foreign bodies in the maxillary synus: between malpraxis and medico-legal consequences. Rom J Leg Med (23) 2015, 14-18.

11. Pynn B.R., Nish I.A., ORAL SURGERY: Maxillary Sinusitis: A Review for the Dental Practitioner, Oral Health, May 1, 2001.

12. Dobele I, Kragis G .Sinusitis of Odontogenic Origin: Implications of Clinical and Radiological Diagnostics. Ann Otolaryngol Rhinol 3(4): 1099. (2016).

13. Shenoy V, Maller V, Maller V. Maxillary Antrolith: A Rare Cause of the Recurrent Sinusitis. Otolaryngology Volume 2013, Article ID 527152.

14. Haraji A., Mohtasham N., Zareh MR., Pezeshkirad H. Antrolith in the Maxillary Sinus; Report of a Case. Journal of Dentistry, Tehran University of Medical Sciences, Tehran, Iran 2006; Vol: 3, No.2.

15. KANLIS A, KARAGÜL E, AKKAYA N, ÖZBEK M. Maxillary Antrolith: A Case Report . Hacettepe Diş Hekimliği Fakültesi Dergisi Cilt: 32, Sayı: 4, Sayfa: 103-106, 2008.

16. Han $\mathrm{MH}$, Chang $\mathrm{KH}$, Lee $\mathrm{CH}$, Na DG, Yeon KM, Han MC. Cystic Expansile Masses of the Maxilla: Differential Diagnosis with CT and MR. AJNR Am J Neuroradiol 16:333-338, February 1995.

17. Gonçales ES, Guedes A ,Gonçales B , da Silva Lima E, Rocha JF, Noleto JW , Hochuli-Vieira E. Symptomatic mucous retention cysts of the maxillary sinus: case report RSBO. 2015 AprJun;12(2):233-237.

18. Giotakis El, Weber RK. Cysts of the maxillary sinus: a literature review. Int Forum Allergy Rhinol. 2013;3:766-771

19. Paulino A, Marks JE, Bricker P, Melian E, Reddy SP., Emami B, Results of Treatment of Patients with Maxillary Sinus Carcinoma.CANCER August 1, 1998 / Volume 83 / Number 3,457465. 
20. Blanch JL, Ruiz AM., Alos L, Traserra-Coderch J, Bernal-Sprekelsen M.Treatment of 125 Sinonasal Tumors: Prognostic Factors, Outcome, and Follow-up.Otolaryngol Head Neck Surg December 2004 vol.131 no.6973-976

21. Mehanna P., Smith G. Maxillary carcinoma. A wolf in sheep's clothing. Can Fam Physician. 2009 Mar; 55(3): 262-264.

\title{
Corresponding author:
}

\author{
Elitsa Deliverska \\ Sofia 1431 \\ Str. Georgi Sofiiski 1 \\ Department of Oral and Maxillofacial surgery, \\ Faculty of Dental medicine \\ Medical university- Sofia
}

